\title{
The Mind's Room Project : A Model Case of Interdisciplinary Cooperation
}

\author{
Masaharu Mizumoto* and Masato IsHikawa**
}

\begin{abstract}
In Mizumoto \& Ishikawa (2002), we introduced a paradigm of experiment which is of interest to both psychology as well as philosophy. This experiment involved a subject wearing a Head-Mount Display (HMD) while a camera was set up in the upper corner of the same room in which the subject was to be found. In the experiment images were sent wirelessly from the camera to the HMD, so that the subject could observe himself from the third-person perspective through the HMD. In this paper we will discuss the interdisciplinary aspect of this experiment, presenting specific results of psychological experiments as examples together with outlines for further experiments.
\end{abstract}

\section{Introduction}

The Mind's Room Project is a paradigm of experiment in which the subject wears a goggle-style monitor, or HMD (Head-Mount Display), while a camera is set up in the upper corner of the same room (henceforth to be called the Mind's Room) in which the subject finds himself. As a result of visual information being sent wirelessly from the camera to the HMD the subject is capable of observing his own body through this monitor, creating for the subject a quasi disembodied sense "My body is there!" (Figure 1)

The idea behind the setting of this experiment was first conceived of within the context of philosophical thought experiments concerning the traditional problem of body and self. ${ }^{1}$

*School of Arts and Letters, Meiji University.

**School of Information and Communication, Meiji University

1 One may find a similar but more sensory-oriented thought experiment in Dennett (1978), while a much more direct ancestor of the present idea may be found in Wittgenstein's RPPI 753 (though Mizumoto found this passage after he had independently conceived of the idea himself). See Mizumoto (2002) for the interpretation and discussion of this passage. One of the referees of this journal pointed out the implication for and connection with Gallup's famous mark test (1970), which allegedly showed that chimpanzees could recognize their own images in a mirror as themselves. The same referee also suggested the relevance of this experiment to the work of visual transposition experiments undertaken by Kaoru Sekiyama. Mizumoto has indeed studied Sekiyama (1997) and other work on visual transposition by Hirokazu Yo- 


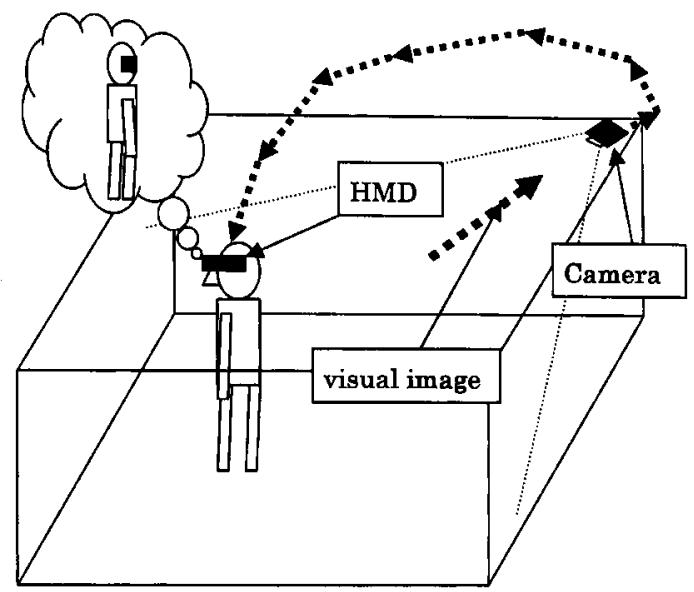

Figure 1: Paradigm of the Mind's Room

\section{Preliminary Experiment}

After conducting several preliminary experiments, including long-term (three day) experiments, several interesting facts have come to light. ${ }^{2}$

The first thing one finds in this experimental situation is no doubt the awkwardness and difficulty for the subject in moving around the room. It is certainly an extremely abnormal situation to have one's perspective located outside of one's body as well as being able to watch it from another perspective in real time. Furthermore, since what we see through the HMD when we face the camera is exactly the reverse image of what we usually see of ourselves in a mirror, the coordination between the (non-visual) body image and the visual image of the body is dissociated in an unusual manner, making it feel all the more difficult to control one's body in this situation. Because of this, some subjects reported that they felt as if they had "regained" their body after they closed their eyes at the time confusion occurred.

One of the authors himself participated in the long-term experiment, and in performing various tasks designed to help one become adjusted to the space (for example toying with a balloon), came up with the following mental map of the Mind's Room.

As can be seen (Figure 2), since the left and right-hand sides for the camera coincide with the left and right-hand sides of the body, by turning his back to the camera the author in question could reduce the feelings of difficulty experienced when coping with the space inside of the room. What is interesting about this observation is that keeping this kind of cognitive map in mind reduced confusion

shimura, though we unfortunately could not discuss them in this paper. We would like to thank the referee for pointing out these works.

2 The following discussions are mainly based on Mizumoto \& Ishikawa (2002). 


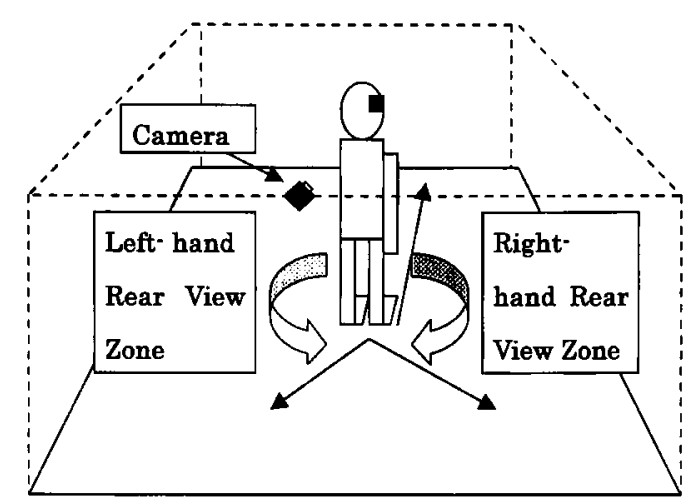

Figure 2 : Cognitive Map of the Mind's Room (1)

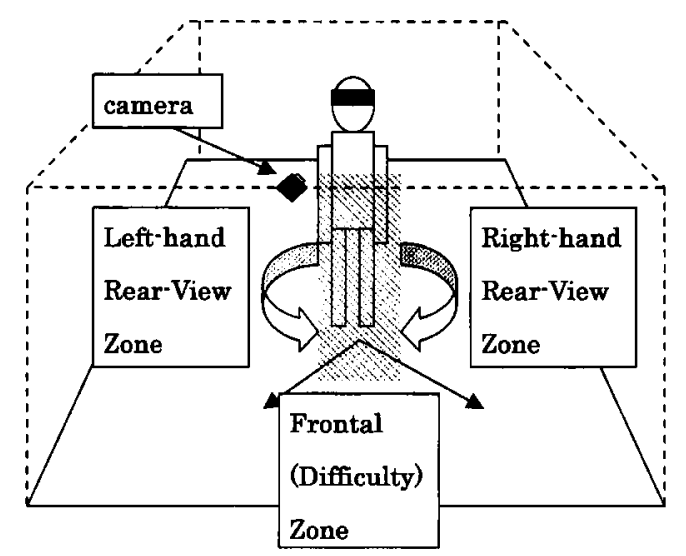

Figure 3: Cognitive Map of the Mind's Room (2)

even while the subject was facing the camera. (Figure 3)

That is, whereas the frontal space (where one's right hand appears on the left from the camera) continued to pose a number of difficulties for the subject when performing actions such as striking a balloon, when we imagined as if we had turned our back on the camera, and thereby regarded the left-hand side of the space (as seen from the camera) as the space into which we would "look back" when looking over the left shoulder if our back were turned to the camera, and the right-hand side (as seen from the camera) in the same fashion, over the right shoulder, confusion that could arise when say, attempting to strike a balloon in the air (for example using the wrong, i.e. opposite, hand) consequently reduced considerably, even if the body was actually still facing the camera (though this observation is based solely on verbal reports).

In one experiment in the Mind's Room, which we will call the Bodily Illusion Experiment, two subjects wearing HMDs in the same room and sharing the same visual image and space, are asked to sit on chairs set next to each other. The chairs 


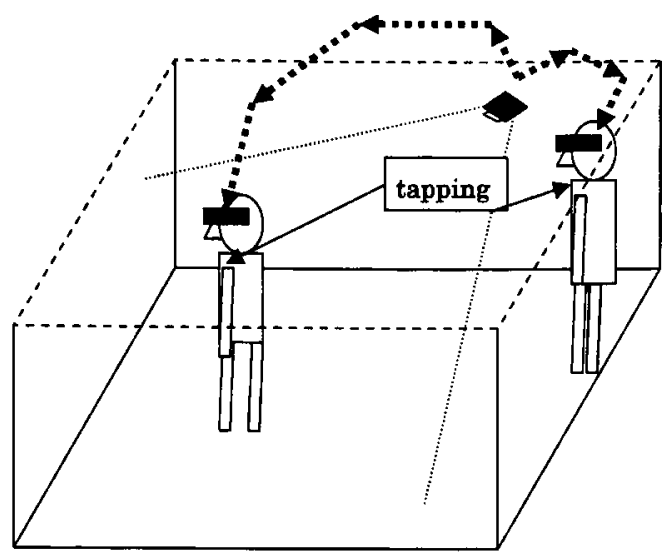

Figure 4: Bodily Illusion Experiment (Note that although in this figure subjects are depicted as standing, the structure of the experiment is essentially the same.)

are facing the camera, however one is set outside the visual frame of the camera (thus outside that of the HMDs as well), while the other is within this frame, so that when sitting, only one of the two subjects is visible to both of them. Following this, an experimenter taps simultaneously on both the subjects' (for example) left shoulder, at exactly the same (but irregular) pace. (Figure 4)

The subjects whose body was outside the visual frame of the HMD, watching the other subject being tapped on the shoulder, unanimously (all four subjects who participated in this particular experiment) reported that they 'felt' as if the body they were watching were their own, although they knew in fact this not to be the case. This seems to suggest that through the tapping sensation, the proprioceptive self of a person can be misidentified with the body of another person.

The difference between the above and the more easily possible instance of misidentifying oneself in a mirror is the strong belief-independence of this "feeling." To demonstrate this, during the tapping experiment another experimenter pretended to suddenly hit the subject, whose body was within the visual frame of the camera, on the head. The idea behind this act was that if the subject who was outside the visual frame of the camera yet who was conceivably viewing an illusion of his own body reacted ('mistakenly') to the attack, then he would be shown to be truly feeling as if the body depicted on his monitor were his own, despite the actual fact that it was not really his own body. In effect, we did indeed succeed in videotaping just one such subject, who, just after reporting the expected bodily illusion, reacted to the sudden (mock) attack, which was actually an attack on the other subject. ${ }^{3}$

${ }^{3}$ See Mizumoto \& Ishikawa (2005) for the details of this experiment and its philosophical implications. 


\section{One Application}

Since the reasearchers who conducted the last experiment were not trained in psychology, the experiments above were conducted largely in an informal manner. In other words, in order to make a true scientific contribution the project still required some form of collaboration with specialists within the field. Thus we held a campaign to promote the project as well as inform others of it through presentations and demonstrations in various psychology seminars and meetings - in fact we were often invited to do so. However, though many psychologists we met expressed interest in our project, few specific plans of experiment were suggested at first. We believe that this is due to the fact that unlike the habitual course of scientific research, whereby scientists devise and conduct new experiments based on some preceding findings published or reported, in our case we begin by first introducing a mere framework of experimentation which scientists must then 'interpret' and locate within the context of their own current research. This is a kind of hermeneutic process, which, though not explicit in normal scientific activities, nonetheless underlies, and is even essential to, any science.

In spite of such difficulty, several proposals were made and are now in progress. One such experiment has already been conducted and we have been able to obtain a specific result, which we will discuss presently.

In research concerned with scene recognition across view changes, it has come to be known that as we move in an environment our personal representation of a given scene is "updated," even though we do not directly observe that scene during the period of time in which the change in viewpoint occurs. This fact has been demonstrated by change detection tests in which subjects are asked what differences they might notice with regard to a given arrangement of objects after a change in orientation (rotation of the display of objects) or after a change in viewpoint (movement of the subject) was made, with evidence indicating that subjects' performance in the latter was suprerior to their performance in the former. (Simons \& Wang, 1998)

Also known are the facts that not only can subjects update this mental representation even if they are moving in the dark and cannot see (Simons \& Wang, 1998), but that the same result can be obtained as well even when the subject's movement is merely visual-that is, when subjects are provided with a virtual image of movement which can be distinguished visually from the mere rotation of the display of objects (Yoshino \& Kitazaki, 2002 and Kayahara et. al., 2003).

One of the authors (Mizumoto) together with psychology student Junichi Hasegawa, Chuo University, applied the framework of the Mind's Room experiment to the work done in this field and conducted the following experiment. 


\section{Experiment 1 and 2}

\section{Method}

Subjects: 10 undergraduate students ( 5 males, 5 females)

\section{Materials and Procedure}

An HMD and a camera that sends visual images to the HMD. The camera is directed towards a circular table, in front of which a subject sits on a chair on castors, enabling it to be moved around the table. As well, the table itself can be rotated (see Figure 5). The camera is first set up next to the chair (Experiment 1) and then behind the chair (Experiment 2). The camera can be moved around the table just as the chair. In Experiment 1 the camera captures only the table and its background, while in Experiment 2 it includes the subject's body sitting on the chair within the frame as well.

Subjects were given the following instructions; they were asked to memorize the arrangement of 5 objects (a stone, a stapler, scissors, a cup, and a toothbrush) which were placed upon the table within 3 seconds. After 3 seconds, the table was covered from the subjects, and while it was covered, the camera and the chair (in conditions $\mathrm{B}$ and $\mathrm{C}$ ) were moved 40 degrees around the table and the experimenter changed the position of one of the five objects that had been placed on the table (in 7 seconds). Subjects did not know in advance the direction in which their chair would be moved, if moved at all. After the cover was removed the subjects were asked if they could tell which object had been moved. The table was also rotated 40 degrees for half of the trials.

Conditions of each trial were controlled by the three types of information of the movement and whether or not the retinal image of the display changed or remained the same by the combination of the rotation of the table and the movement of the camera (thus for Experiment 1 and 2, $3 \times 2 \times 2(=12)$ conditions in total). Each subject went through all the conditions, 5 trials for each.

One type of information received by subjects concerning movement was merely visual (A), where the camera (but not the chair) was moved around the table 40

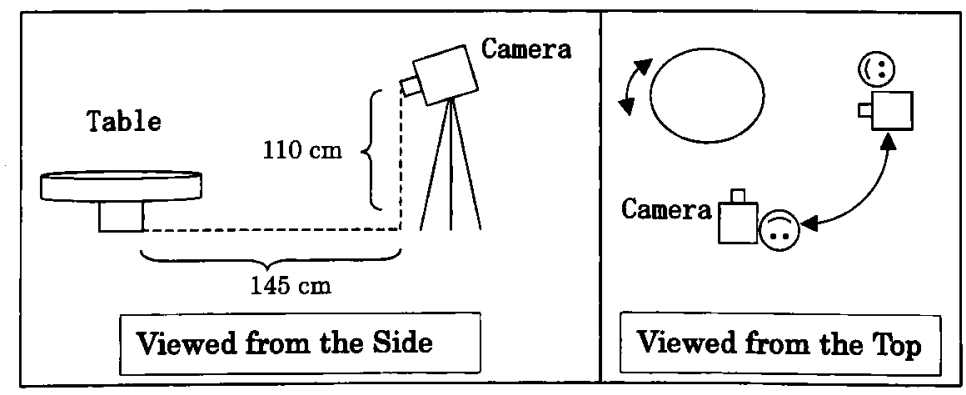

Figure 5 


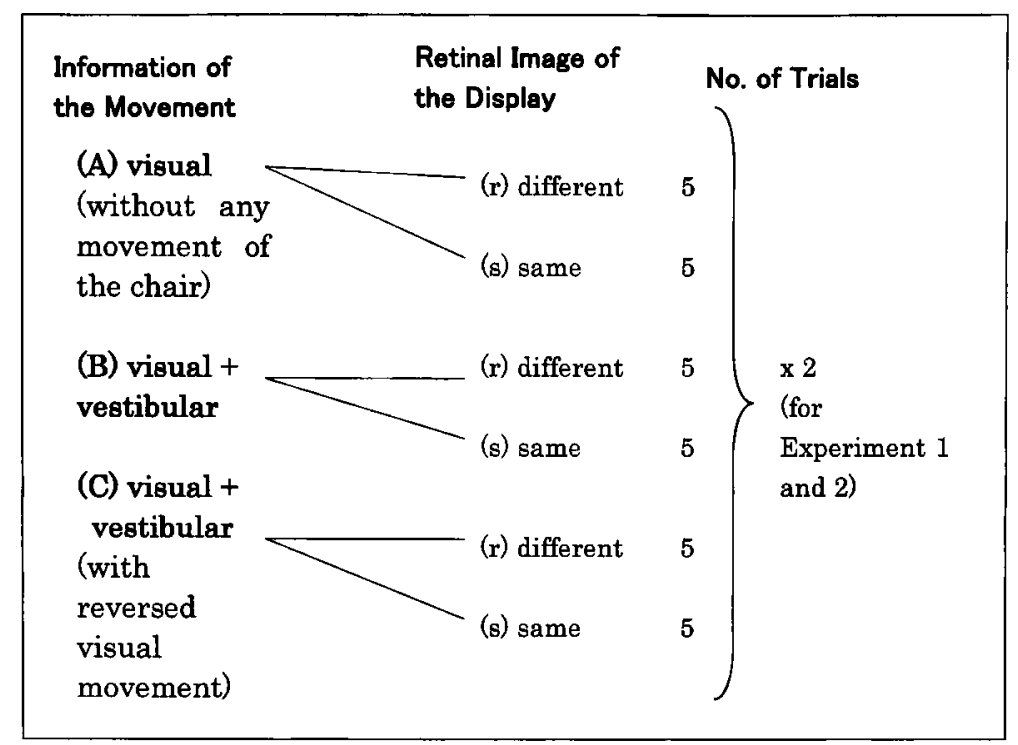

Table 1

degrees. Other types were a mixture of visual and vestibular information, one with 40 degrees of movement of both the camera and the chair in the same direction (B), the other with the same movement involving the camera and the chair, however this time a mirror was set up before the camera, providing it with the image of reversed movement (C). Note that, therefore, in every condition the camera was moved while the chair was moved only in $\mathrm{B}$ and $\mathrm{C}$, and the table was rotated on half of the trials in each condition.

The retinal image of the display of the objects was changed for subjects when the table was fixed while the camera was moved ( $r$ ), and remained the same when the table was rotated 40 degrees in the same direction as the camera (s).

\section{Results and Discussion}

The results of these experiments are shown in tables 2 and 3 below.

As we can see, in both experiments subjects remembered the arrangement of objects better when the retinal image changed rather than when it remained the same while the camera moved. This was the case whether there was vestibular information concerning the movement or not, confirming a result that has already been reported (in Yoshino \& Kitazaki, 2002 and Kayahara et. al., 2003). That is, mere visual information of movement (when the body does not move) is enough to update the representation of the relevant environment.

In condition (C), where there is a discrepancy between the visual and the vestibular information about the movement, there was no difference in performance between (r) and (s). This means that even though the vestibular information is not 


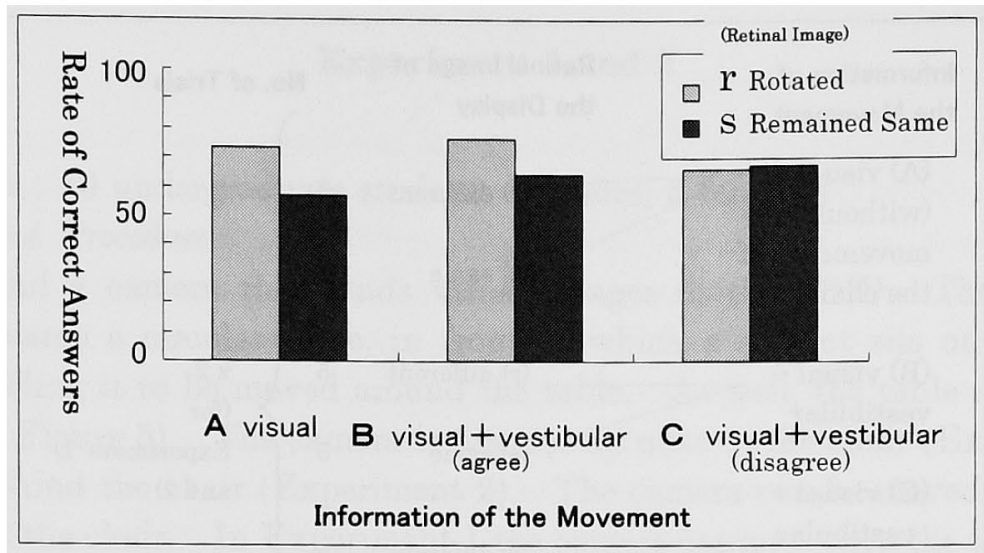

Table 2: Experiment 1

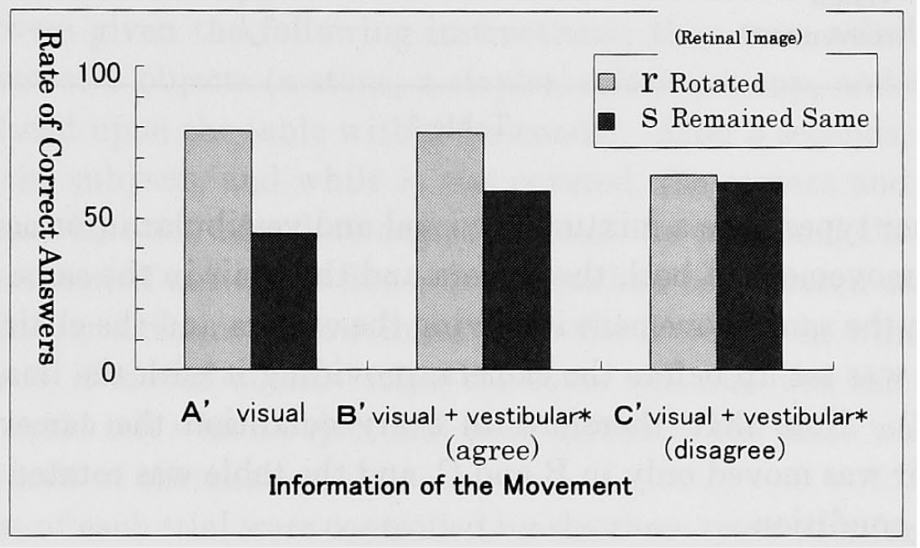

Table 3: Experiment 2

*In $\mathrm{B}^{\prime}$ and $\mathrm{C}^{\prime}$ subjects could see their own body.

necessary for an update of the representation, it does affect the update when it does not coincide with the visual information. Thus the vestibular information has some role in the process of updating the representation, if only a negative one.

In the present experiments we did not observe a significant difference in the results between Experiments 1 and 2, which suggests that though in Experiment 2 clues to tell in which direction the chair was being moved in relation to the table were available to subjects, that information did not help in updating the representation. This fact can be contrasted with the above observation, in which the vestibular information about the direction of the chair did affect the update, where the image was reversed through the use of a mirror (condition $\mathrm{C}$ ), whereas this result tells that the visual information concerning their own movement in relation to the table, did not make difference to the update. One may question here why such information (concerning the location of oneself relative to the table) matters, since it seems 
irrelevant to the present task of remembering the spatial arrangement of objects. However, the same thing could be said about condition $\mathrm{C}$ in general, where no relevant information (which was present in condition A) was missing, so that the subject should have been able to concentrate on only the visual information and ignore the vestibular information about one's own movement.

Then why did the bottom-up information obtained through the vestibular system (negatively) affect the subject's performance in the task, whereas the topdown information available in Experiment 2 neither enhanced nor disturbed the subject's performance? The most plausible answer is that the latter type of information is "too sophisticated" for the task to make a difference, which could be said of any tasks in the Mind's Room Project in general, as well as of any visual transposition experiments. We know, for example, that our right hand is visually placed on the left-hand side, and the left hand is on the right-hand side, yet that "correct" information seems hardly available in the simplest tasks, like striking a balloon. What is needed is some training through which the subject would (or might) regain the coordination between visual and proprioceptive information at the sub-conscious level. Whether such adaptation is possible at all and whether it affects the performance in this task remains an open question, one left for further experiments.

\section{Interdisciplinary Issues and Other Ongoing Projects}

Unlike other ongoing experiments, which we will see below, the experiment in the last section may not ressemble our original Mind's Room experiment, in that the camera was not fixed but moved during the experiment, and in Experiment 1 the subject's body did not even appear in the monitor of the HMD. However, note that (1) the use of an HMD is essential in this experiment, since it made condition $\mathrm{C}$ (the use of a mirror) possible, and (2) the idea for Experiment 2 was a direct application of the setting of the Mind's Room Project.

Yet what we wish to emphasize here is not the superficial similarity of the experimental settings, but rather how the idea of the last experiment came to be conceived of at all. Mizumoto and Ishikawa, one a philosopher and the other an information engineer, first conducted the original Mind's Room experiment and, as already mentioned, later presented and demonstrated the experiment at various meetings of psychologists. However, it was Hasegawa, a psychology student, who interpreted and placed the setting in the context of the scene recognition experiments, and proposed the basic structure for the experiment in the last section. ${ }^{4}$ As

${ }^{4}$ To be more specific about respective contributions, Mizumoto and Ishikawa provided the necessary equipments (HMD, camera, etc.) for Hasegawa, who conducted the 
has been pointed out, though conceiving of a new experiment in this way is uncommon in psychology (and science in general), it would have been very difficult for Hasegawa to conceive of the same experiment completely independent of the Mind's Room experiment.

This can also be said about another psychological experiment under preparation. It is well-known in psychology that subjects are more proficient at recalling or recognizing simple gestures like the act of putting one's hand on one's head when they have actually engaged in learning them (SPT, or subject-performed task), rather than when they had simply read or listened to explanations about them (VT, or verbal task). ${ }^{5}$ The experiment being suggested involves subjects, in the Mind's Room situation, performing (1) SPTs with an HMD and (2) without an HMD, as well as (3) EPTs, or experimenter-performed tasks, with (or without) an HMD.

In condition (3) subjects see a third person enacting the verbally presented instructions. In condition (1) the subjects see themselves enacting them. So condition ( 1 ) is the intermediary condition between conditions (2) and (3), with subjects themselves enacting the instructions, while observing them from the thirdperson perspective. To this may also be added the mirror condition, which provides (through the HMD) subjects with the mirror image of themselves, the reversed image of the normal Mind's Room condition, by placing a mirror just before the camera.

Although we refrain from discussing the details of what can be expected from such experiments at this moment, it is already clear that such experiments factorize the relevant parameters of the SPT effect, which otherwise would not have been so easy.

Yet another experiment is related to the last one. In the original Mind's Room experiment we asked subjects to write some letters or shapes in the air with one of their hands while facing the camera (this is called "Kusho" in Japanese). ${ }^{6}$ Subjects sometimes wrote letters facing the camera, but most of the times they wrote letters facing their own body. Now it is known that this type of writing (Kusho) enhances the results of memory tasks; subjects remember nonsense syllables or shapes better when they write them in the air, or on a piece of paper without a pen, when memorizing. ${ }^{7}$ Thus if one knows about such results it is quite natural to conceive

experiment. Mizumoto also suggested the use of a mirror, however the analysis of data and the interpretation of the results are largely due to Hasegawa.

5 e.g., see Cohen (1989) and Engelkamp \& Zimmer (1989).

${ }^{6}$ It has been reported that the Japanese and the Chinese, who conventionally use Chinese characters in writing, use Kusho voluntarily in memorizing letters or solving specific puzzles related to the structure of Chinese characters. (The same behavior was not observed in those who do not use Chinese characters when solving the same puzzles.) It is even observed that Chinese character users' performance of solving the puzzles are disrupted when Kusho is prohibited. See Sasali \& Watanabe $(1983,1984)$.

7 See Naka \& Takazawa (1990) and Naka (1998). 
of an application of this experiment to something resembling the Mind's Room situation, whereby one would ask a subject to memorize some shapes or syllables by (1) writing them while closing one's eyes, (2) opening one's eyes and writing towards the camera, (3) doing the same thing, but with a mirror set up before the camera, so that the image one sees through the HMD would be the reversed (mirror) image. It can be expected that conditions (1) and (3) might be easier for the subject than condition (2), leading to the corresponding order of the results of memory tests (free recall or recognition).

This experiment would factorize the elements for the memory task in Kusho experiments, and furthermore show which element is relevant in enhancing memory ; visual experience or motor control experience. Now similar to other experiments that have already been considered, it would have been very difficult to conceive of the details of this experiment independently of our project.

Though the Mind's Room Project emerged from a purely philosophical curiosity, it has turned out that it can provide a rich source of inspiration for new experiments in empirical science. Such examples suggest that our project can be seen as a model case of interdisciplinary cooperation between philosophy and empirical sciences.

Now one of the famous and successful examples of philosophical contribution to psychology was Daniel Dennett's False-Belief Task (Dennett, 1978a). This particular proposal of framework of experiment led to a series of intense studies and debates on the "theory of mind" after Baron-Cohen's application of that framework to experiments involving autistic children (Baron-Cohen \& Leslie \& Frith, 1985).

It seems, however, the success of Dennett's proposal depended on very particular empirical conditions (which is to some extent good luck), and was limited to a specific area of psychology. In contrast to this, although our project requires a certain hermeneutic process on the part of scientists in order to conceive of specific applications for it, good applications seem to be found in a wide range of specialized sciences, not only in psychology but also in, e.g., linguistics, social psychology, and (arguably) neuroscience. The success of this project, therefore, does not depend on results of specific experiments ; rather, it should be evaluated on the basis of how many successful applications can be derived from it. ${ }^{8}$

In this connection we may note that Dennett once mocked the general attitudes

${ }^{8}$ Note that there are also technology-driven experiments, where the introduction of new technology makes a new framework of experiment possible. A typical example is the virtual reality equipment. Recent examples are the MVL (multimedia virtual laboratory) project in Japan and VENLab (virtual environment navigation laboratory) in the USA. Admittedly, the Mind's Room Project was also made possible partly by new technology, but it differs from these large-scale projects in that it emerged from a purely philosophical interest, outside of any interests of engineers or empirical scientists. Being free from such specific practical interests is one of the virtues of our project, allowing wider applicability. 
in philosophy of science, which relied mainly on the conceptual analysis of scientific terms, calling such a role a "conceptual policeman." (Dennett 1994, p. 262) He even lamented the shame that it would be, were that to be the main job of philosophy. In response to Dennett and in trying to answer what kind of role philosophy of mind can play in cognitive science qua mind science, or the role of philosophy in science in general, Mizumoto (2004) suggested that the role philosophers can play (and are indeed playing) in specialized sciences can be put as a "conceptual engineer," who not only analyzes concepts but also sometimes proposes a new picture, a new framework of thought or experimentation, a bold hypothesis, a new approach to problem solving, etc., which are often independent of, and therefore alien to, the routine activities in empirical investigations and are indeed wanted by scientists. Thus this conception of the role of philosophical activities assigns to philosophy a role through which it can make a positive contribution to empirical science. The Mind's Room Project is meant to be, and we have argued is, just such an attempt. ${ }^{9}$

\section{Conclusion}

Promoting this project, however, we are not making any specific empirical claim, much less propounding any philosophical thesis other than the metaphilosophical claims just discussed above. Nevertheless, if successful applications accumulate, the empirical results will further reveal the whole nature of this project in general, which would surely be of philosophical interest as well, as it would lend insight into such various topics as self, body, personal identity, ego-centric space and spatial character of vision, cross-modality, affordance, etc., etc.

${ }^{9}$ One of the referees of this journal claimed that it is not clear how Mizumoto's being a philosopher contributed to this cooperation. What he means, we imagine, is that a non-philosopher could have come up with the same idea, and if that is the only basis for the interdisciplinary claim here, then the claim seems somewhat overstated. We agree. What the referee overlooked, however, is that Mizumoto also has independent philosophical interests, like the problem of consciousness, self-identity, and other issues noted in the conclusion, and how much philosophically interesting results the experiments can provide is an empirical issue, just as in the case of Dennett's False-Belief Task (though, as we have noted above, the success of this experiment should also depend on the number of successful applications). In any case, we have emphasized in the conclusion that philosophy as well would also benefit from this experiment, and that alone should be enough for the claim of the significance of interdisciplinary cooperation in this instance (and see for such an example Mizumoto \& Ishikawa 2005). Anyone who wishes to downplay the interest of the present project must form their arguments on an a priori basis, which is, to say the least, not a very fruitful attitude, especially for people with a naturalistic spirit. 


\section{Acknowledgments}

We would like to thank Hirokazu Yoshimura (Housei University), Makiko Naka (Hokkaido University) and Nobu Shirai (Chuo University), for helpful comments and advice. This paper was partly supported by Grant-in-Aid for JSPS Fellows.

\section{References}

Baron-Cohen, S., A. Leslie, and U. Frith. 1985. Does the autistic child have a "theory of mind"? Cognition, $21: 37-46$.

Cohen, R.L. 1989. Memory for action events: The power of enactment, Educational Psychology Review 1, pp. 57-80.

Dennett, D. 1978. Where am I ?, Brainstorms, Cambridge : MIT Press/A Bradford Book.

Dennett, D. 1978a. Beliefs about beliefs, Behavioural and Brain Sciences, 1, 568-70.

Dennett, D. 1994. Artificial Life as Philosophy, Artificial Life 1. 291-2.

Engelkamp, J., \& Zimmer, H.D. 1989. Memory for action events : A new field of research, Psychological Research, 51, pp. 153-157.

Engelkamp, J., \& Zimmer, H.D. 1997. Sensory factors in memory for subject-performed tasks, Acta Psychologica, 96, 43-60.

Gallup, G.G.Jr. 1970. Chimpanzees: Self-recognition, Science.

Hasegawa, J. 2004. The effect of viewpoint change on the scene recognition (in Japanese), Graduate Thesis submitted to Chuo University.

Ishikawa, M. \& Watanabe, T. (ed.) (2004. Maindo Saiensu no Sisou (The Thought of Mind Science) [in Japanese], Tokyo: Shin You Sha.

Kayahara, T., \& Ogi, T., \& Kitazaki, M., \& Yoshino, T., Hirose, M. 2003. Networked Psychological Experiment Systems with Multimedia Virtual Laboratory (MVL) (in Japanese), TVRSJ, Vol. 8, No. 1, pp. 57-64.

Mizumoto, M. 2002. Intention, Body, and Vision Wittgenstein's 'Experiment', Kagaku Tetsugaku (Philosophy of Science): Journal of the Philosophy of Science Society, Japan, 35-1, pp. 27-42 (in Japanese with English abstract).

Mizumoto, M. \& Ishikawa, M. 2002. Mind's Room Project (in Japanese), Journal of the Japan Association for Philosophy of Science, No. 98, pp. 29-35.

Mizumoto, M. 2004. Chap. 3 : Philosophy of Mind, in Ishikawa \& Watanabe (2004).

Mizumoto, M. \& Ishikawa, M. 2005. Immunity Thesis and the Bodily Illusion Experiment, The Journal of Consciousness Studies, Vol. 12, No. 7. pp. 3-19.

Naka, M. and Takizawa, M. 1990. Writing over and over to remember? Then why? Chiba Daigaku Kyouiku Gakubu Kiyou, 38(1), pp. 31-36.

Naka, M. 1998. Repeated writing facilitates children's memory for pseudocharacters and foreign letters, Memory \& Cognition, 26(4), pp. 804-9.

Sasaki, M. \& Watanabe, A. 1983. An experimental study of spontaneous writinglike behaviour ("Kusho") in Japanese (in Japanese), Japanese Joumal of Educational Psychology, Vol. XXXI, No. 4, pp. 273-282.

Sasaki, M. \& Watanabe, A. 1984. Cultural origin of "Kusho" behaviour, Japanese Journal 
of Educational Psychology, Vol. XXXII, No. 3, pp. 182-190.

Sekiyama, K. 1997. Shintai Hyoushou to Kuukan Ninchi (The body representation and Spatial Recognition) Tokyo: Nakanishiya.

Wittgenstein, L. 1980. Remarks on the Philosophy of Psychology I (RPPI), Oxford : Basil Blackwell.

Yoshino, T. \& Kitazaki, M. 2002. The effect of the viewpoint changes on scene recognition in the space of virtual reality (in Japanese), TVRSJ, Vol. 7, pp. 75-8. 